\title{
Stress dissipation in cucurbit[8]uril ternary complex small molecule adhesives
}

\author{
Paul E. Williams, ${ }^{\dagger, \ddagger}$ Zarah Walsh-Korb, ${ }^{,+,+,}$, Samuel T. Jones, $^{\dagger}$ Yang Lan, ${ }^{\dagger}$ and \\ Oren A. Scherman ${ }^{*, \dagger}$ \\ $\dagger$ Melville Laboratory for Polymer Synthesis, Department of Chemistry, University of \\ Cambridge, Lensfield Road, Cambridge, CB2 1EW, United Kingdom \\ $\ddagger$ Both authors contributed equally to this work \\ ФBioTeam, ECPM-ICPEES, UMR 7515, Université de Strasbourg, 25 rue Becquerel, 67087 \\ Strasbourg, Cedex 2, France \\ E-mail: walshkorb@unistra.fr; oas23@cam.ac.uk
}

Phone: +33 (0)3 688527 07; +44 (0)1223 331797. Fax: +33 (0)3 688527 16; +44

(0)1223 336488

\begin{abstract}
The ability to control supramolecular and macroscopic self-assembly and disassembly holds great potential for responsive, reversible adhesives that can efficiently broker stresses accumulated between two surfaces. Here, cucurbit[8]uril is used to directly adhere two functionalized mica substrates creating surface-surface interactions that are held together through photo-reversible $\mathrm{CB}[8]$ heteroternary complexes. Comparison of single molecule, bulk and macroscopic adhesion behavior give insight into cooperativity and stress dissipation in dynamic adhesive systems.
\end{abstract}

Mimicking Nature to gain external or remote control over the interactions between two surfaces is attractive and has engaged many researchers over the past decades. ${ }^{1,2}$ Maintaining 
a truly reversible adhesive process, however, has long been an obstacle to achieving this goal. Many applications including switchable circuitry, robotics, sensor design and tissue adhesion in the biomedical sphere ${ }^{1-4}$ require controllable adhesion at the solid/solid interface, adding additional complexity to the problem.

Macrocyclic host molecules such as cyclodextrins (CDs) and cucurbit[n]urils (CB[n]s, specifically where $n=7$ or 8 ) have been shown to promote adhesion between a variety of substrates at nanometer-micron scale with a combination of chemical, electrochemical and photochemical controls across liquid/air and liquid/solid interfaces. ${ }^{5-16}$ Recent work by Harada et al. and Kim et al. have demonstrated the ability of these macrocycles to control adhesive interactions between two solid substrates. ${ }^{17-23}$ However, in these systems, the host molecules are attached to polymer chains which make up the bulk of the solid interface. Thus, two obstacles exist in fully understanding adhesive interactions in these systems: (i) polymer entanglement after relaxation cannot be separated from the contribution of the host-guest interactions, and (ii) the host cannot be removed from the system to eliminate adhesion. In order to address these issues and independently probe host-guest interactions, removing them from polymer entanglement, an adhesive system based on small molecules alone is required.

We have designed a small molecule system depicted in Figure 1, where one surface is functionalized with a silane linked to a methyl viologen derivative and a second surface bears an azobenzene with an identical silane linker.

$\mathrm{CB}[8]$ mediates interactions between these two functional mica surfaces, through the formation of heteroternary complexes. A major advantage of a ternary system over previously published binary host-guest systems (with functionalized CD and CB[7]) is that the dynamic interaction between complementary moieties can be removed to eliminate adhesion, directly addressing obstacle (ii) above. In carrying out experiments on macroscale adhesion between two complementary surfaces linked by CB[8], it was observed that the forces of adhesion obtained did not scale linearly with the single molecule forces obtained from similar systems 


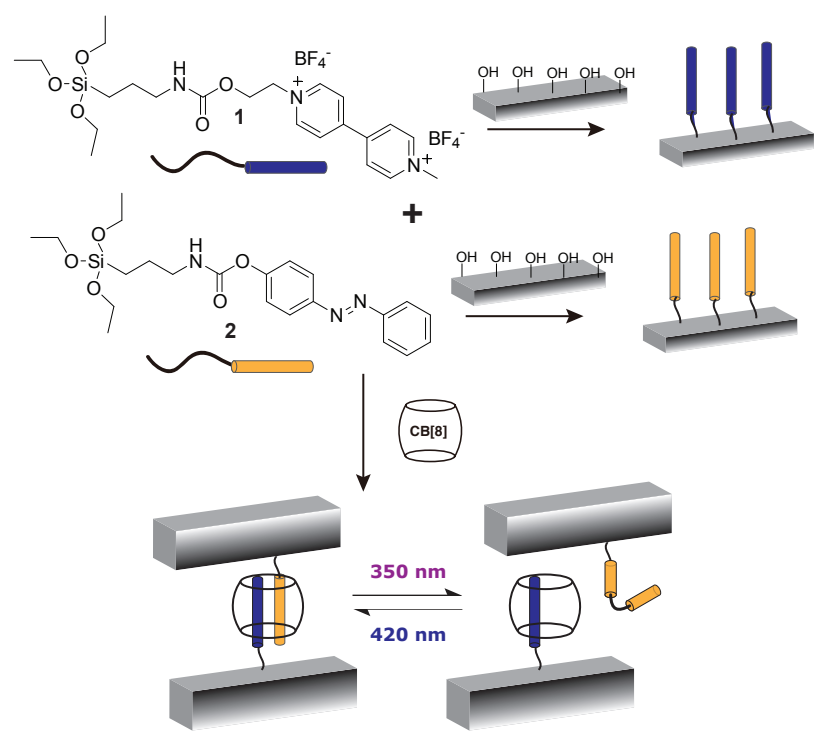

Figure 1: Schematic illustration of the concept and functionalization of photo-reversible surface-surface adhesion mediated by $\mathrm{CB}[8]$ ternary complex formation

in previous studies. In order to better understand the reason for such a discrepancy, the forces of adhesion for the system on the single molecule, bulk and macro-scale were studied for a photo-switchable model $\mathrm{CB}[8]$ ternary complex and the results highlight the role of stress dissipation and cooperativity in dynamic surface-surface adhesion.

Recently, we have developed a method to quantify adhesion forces $\left(\mathrm{F}_{\text {adh }}\right)$ exhibited by the formation of $\mathrm{CB}[8]$ ternary complexes using single molecule force spectroscopy (SMFS). ${ }^{24}$ $\mathrm{F}_{a d h}$ for the formation of a heteroternary complex between a methyl viologen (MV) derivative and naphthol (Np), mediated by $\mathrm{CB}[8]$ was found to be between 140-160 pN. However, such isolated single interactions are not representative of adhesion in the bulk, where cooperativity and stress dissipation across the surface play a large role in the apparent force of adhesion. In order to better understand these phenomena and their contribution to adhesive strength at the interface, mica surfaces were functionalized with silane 1 or $\mathbf{2}$ (Figure 1) as described in the ESI, Section S1. Mica was chosen as the optimal substrate for this investigation on account of its tendency to crack or delaminate if stress is not appropriately dissipated across the entire surface. Here, azobenzene was selected as the second guest to form the heteroternary complex in order to probe reversibility of the adhesive interactions through 
external control, using alternating visible and UV light instead of reduction-oxidation of the surface units, Figure 1. ${ }^{8,25}$

Functionalization of the $\mathrm{MV}^{2+}$ surface and subsequent saline washes led to the formation of mica-1. To confirm the immobilization chemistry did not interfere with the ability of the MV moiety to participate in ternary complex formation, contact angle measurements were carried out to determine the change in the surface contact angle during each step of the functionalization. Both rhodamine-tagged azobenzene (Rh-Azo) and ethylene glycol/naphthol (95/5)-functionalized $20 \mathrm{~nm}$ gold nanoparticles (Np-AuNP) (see synthesis protocol in ESI, Section S1) were used to visualize CB[8] ternary complexation and demonstrate the capability of the mica-1 to interact with both small molecules and larger multi-functional surfaces.

Surface complexation studies were carried out in the presence of $\mathrm{CB}[8]$, and $\mathrm{CB}[7]$ as a control, to demonstrate that heteroternary complex formation only occurs in the presence of $\mathrm{CB}[8]$. No adhesion of $\mathrm{Rh}-\mathrm{Azo}$ or $\mathrm{Np}-\mathrm{AuNP}$ was observed in the presence of $\mathrm{CB}[7]$ as the cavity can only be filled with the MV derivative without any remaining space for a second guest. In all experiments the surfaces are immersed $0.2 \mathrm{mM}$ solution of the macrocycle before extensive rinsing with water to ensure only complexed macrocycles remained on the surface. In all cases the concentration of the $\mathrm{CB}[\mathrm{n}]$ was determined from the weight of the molecule including bound water as determined from X-ray crystallography $(1250 \mathrm{~g} / \mathrm{mol}$ for $\mathrm{CB}[7]$ and $1701 \mathrm{~g} / \mathrm{mol}$ for $\mathrm{CB}[8])$. The resulting contact angle measured at each stage of functionalization is summarized in Table 1 (see also Figure S2 and S3, ESI).

Table 1 shows no change in the contact angle of mica- $\mathbf{1}$ in the presence of $\mathrm{H}_{2} \mathrm{O}$ or aqueous solutions of Rh-Azo and $\mathrm{Np}-\mathrm{AuNP}$ indicating no specific interaction with the functionalized surface. Addition of both $\mathrm{CB}[7]$ and $\mathrm{CB}[8]$ show a decrease in the surface contact angle in correlation with previous observations. ${ }^{7,8}$ Subsequent immersion of the CB[7]-complexed mica-1 into Rh-Azo or Np-AuNP solutions does not produce a change in contact angle. On the other hand, immersion of the $\mathrm{CB}[8]$-complexed mica-1 into $\mathrm{Rh}-\mathrm{Azo}$ produced a large increase in the surface contact angle from the hydrophobic Rh-Azo attached to the surface. 
Table 1: Summary of contact angle changes as a function of surface modification

\begin{tabular}{cl}
\hline Modification & $\mathrm{MV}-\mathbf{1}$ \\
\hline $\mathrm{H}_{2} \mathrm{O}$ & $24^{\circ}$ \\
$\mathrm{Rh}-\mathrm{Azo}$ & $24^{\circ}$ \\
$\mathrm{Np}-\mathrm{AuNP}$ & $24^{\circ}$ \\
$\mathrm{CB}[7]$ & $18^{\circ}$ \\
$\mathrm{CB}[8]$ & $14^{\circ}$ \\
$\mathrm{CB}[7] / \mathrm{Rh}-\mathrm{Azo}$ & $18^{\circ}$ \\
$\mathrm{CB}[8] / \mathrm{Rh}-\mathrm{Azo}$ & $39^{\circ}$ \\
$\mathrm{CB}[7] / \mathrm{Np}-\mathrm{AuNP}$ & $18^{\circ}$ \\
$\mathrm{CB}[8] / \mathrm{Np}-\mathrm{AuNP}$ & $21^{\circ}$ \\
\hline
\end{tabular}

Attachment of $\mathrm{Np}-\mathrm{AuNP}$ also produced a change in the contact angle of $\mathrm{CB}[8]-$-complexed mica- $\mathbf{1}$ but to a lesser extent.

The construction of the heteroternary complexes on the mica-1 with $\mathrm{Np}-\mathrm{AuNP}$ also served a second purpose, to approximate the number of binding sites available. This was carried out by AFM in tapping mode on the mica-1.CB[8]:Np-AuNP surface as well as the control mica-1·CB[7] surface (ESI, Section S5). AFM images of the mica-1.CB[8]·Np-AuNP surface (Figure S4B) illustrate well dispersed nanoparticles across the entire imaged area, while Figure S4A of the mica-1.CB[7] surface shows no dispersed particles. The negative control confirms that it is only possible to adhere the $\mathrm{Np}-\mathrm{AuNP}$ to mica- $\mathbf{1}$ through $\mathrm{CB}[8]$ ternary complexation while $\mathrm{CB}[7]$ prevents surface-NP attachment. The even distribution of the nanoparticles across the mica-1·CB[8] surface enabled a calculation of the MV surface density to be $1 \times 10^{9}$ binding sites $/ \mathrm{mm}^{2}$. The number of available sites to which silane $-\mathbf{1}$ could react on $1 \mathrm{~mm}^{2}$ of mica is $1 \times 10^{14}$, indicating a surface coverage of MV units of only $0.001 \%$, which equates to approximately $1 \mathrm{MV}$ unit every $300 \mathrm{~nm}$ in all directions. The relatively low surface coverage likely arises from the doubly cationic MV moiety and the relatively bulky counterions during surface functionalization (ESI, Section S4). ${ }^{8,24}$

Following confirmation of the surface functionalization, characterization of the adhesive properties at the macroscale was attempted. Figure $2 \mathrm{~A}$ and B illustrate schematically the experiments conducted under visible light $(\lambda=420 \mathrm{~nm})$ and when $0.2 \mathrm{mM} \mathrm{CB}[8]$ was added 
between mica-1 and mica-2, respectively, while Figure $2 \mathrm{C}$ shows the system under UV light $(\lambda=365 \mathrm{~nm})$ in the presence of $\mathrm{CB}[8]$.

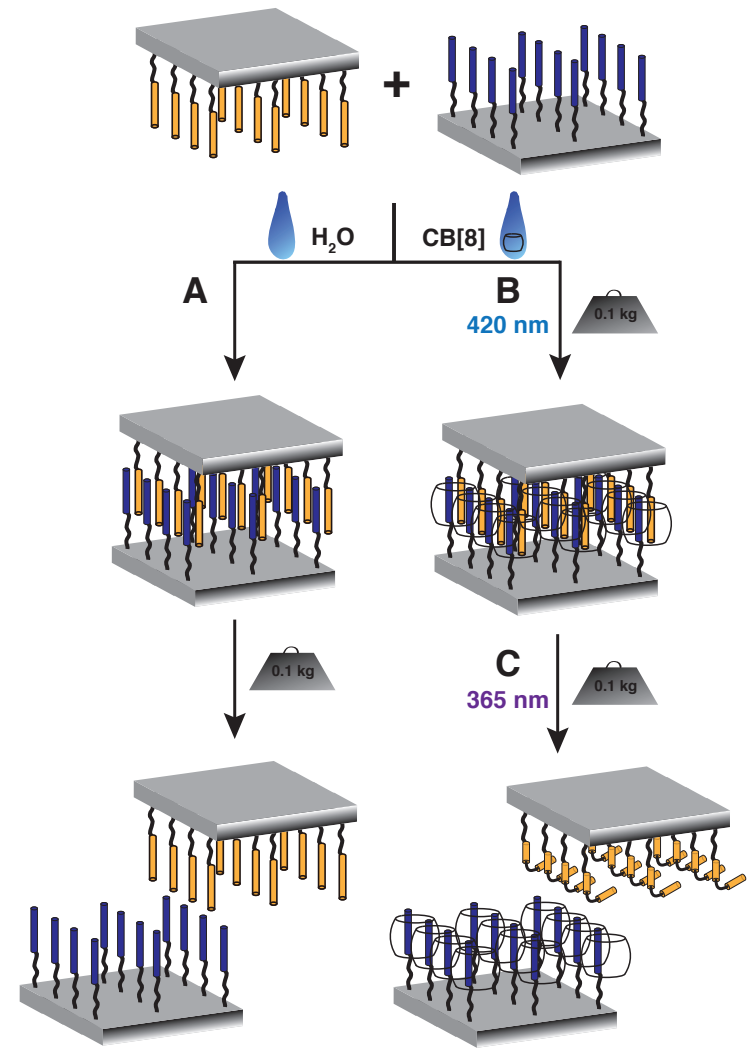

Figure 2: Schematic showing the adhesion of the surfaces in the presence of $(\mathrm{A})$ water and visible light irradiation $(\lambda=420 \mathrm{~nm}),(B)$ a $0.2 \mathrm{mM}$ solution of $\mathrm{CB}[8]$ and visible light irradiation $(\lambda=420 \mathrm{~nm})$ and $(\mathrm{C})$ a $0.2 \mathrm{mM}$ solution of $\mathrm{CB}[8]$ and UV light irradiation $(\lambda=365 \mathrm{~nm})$.

A video of the experiments represented in Figure 2 is available as supporting information. In each case a weight of $100 \mathrm{~g}$ is applied to mica-1 for the duration of the experiment, while the mica-2 is suspended from the inside of a UV-light box. Experiments were conducted after dipping into water or the CB[8] solution and, subsequent pressing of the mica-1 and mica-2 to remove any excess solution. Application of the $100 \mathrm{~g}$ weight in the absence of $\mathrm{CB}[8]$ (Figure 2A) shows no discernible adhesion under visible light irradiation. Introducing a $0.2 \mathrm{mM}$ solution of $\mathrm{CB}[8]$ between the surfaces, results in significant adhesion even when the $100 \mathrm{~g}$ weight is applied (Figure 2B). Under visible light irradiation it is possible 
to hold the $100 \mathrm{~g}$ weight for up to $3 \mathrm{~h}$ without separation of mica-1 and mica-2 or rupture/delamination of the mica layers. Irradiation with UV light $(\lambda=365 \mathrm{~nm})$ induces a trans-cis conversion of the surface-bound azobenzene moieties of mica-2 and subsequent rupture of the ternary complex, destroying the adhesive affect. This process is reversible and repeatable over numerous cycles. These adhesion experiments enabled approximation of $\mathrm{F}_{a d h}$ per available binding site of $1597 \mathrm{pN}$, an increase of almost 20-fold on the $\mathrm{F}_{a d h}$ of $85 \mathrm{pN}$ approximated for the single heteroternary complex as measured by SMFS. The calculated $\mathrm{F}_{\text {adh }}$ of $1.57 \mathrm{kPa}$ over the mica surface $(2.5 \mathrm{~mm} \times 2.5 \mathrm{~mm})$ indicates that the maximum stress, which can be effectively dissipated through the dynamic ternary complexes at the interface, is also in the region of $1.57 \mathrm{kPa}$ otherwise the surfaces would not remain adhered to one another (ESI, Section S3). The calculated $\mathrm{F}_{a d h}$ is approximately the pressure required to break a single standard glass pane by sonic boom, that is the acoustic waves produced by an object moving through air at faster than the speed of sound. ${ }^{26}$

In order to better understand this 20-fold discrepancy between single and multiple CB[8] ternary complex binding events, force-distance spectroscopy (FDS) of bulk tip-surface interactions were performed on the AFM. Similar to the functionalization of mica-2, gold i-Drive AFM cantilevers (Asylum Research) were immersed in a solution of an azobenzene derivative, where the silane linker was replaced by a short alkyl thiol group to facilitate binding to the $\mathrm{Au}$ tip. Figure 3A-C show three control experiments, which determine the extent of the tip-sample interactions without formation of the $\mathrm{CB}[8]$ ternary complex.

In each experiment the tip was brought in contact with the surface 2500 times over an area of $20 \mathrm{~mm} \times 20 \mathrm{~mm}$, with a spacing between points of $400 \mathrm{~nm}$ in both $x$ and $y$ directions. The $\mathrm{F}_{a d h}$ required to remove the unfunctionalized Au-cantilever from the mica-1 is rather high, with the average retraction force in the region of $65 \mathrm{nN}$, Figure $3 \mathrm{~A}$. This is likely due to the strong attraction of the negatively charged Au cantilever with the dicationic $\mathrm{MV}^{2+}$ on the mica-1 surface. When $0.2 \mathrm{mM} \mathrm{CB}[8]$ is added to the system without an azobenzenefunctionalized tip, the $\mathrm{CB}[8]$ binds the $\mathrm{MV}^{2+}$ moieties on mica-1 eliminating the non-specific 

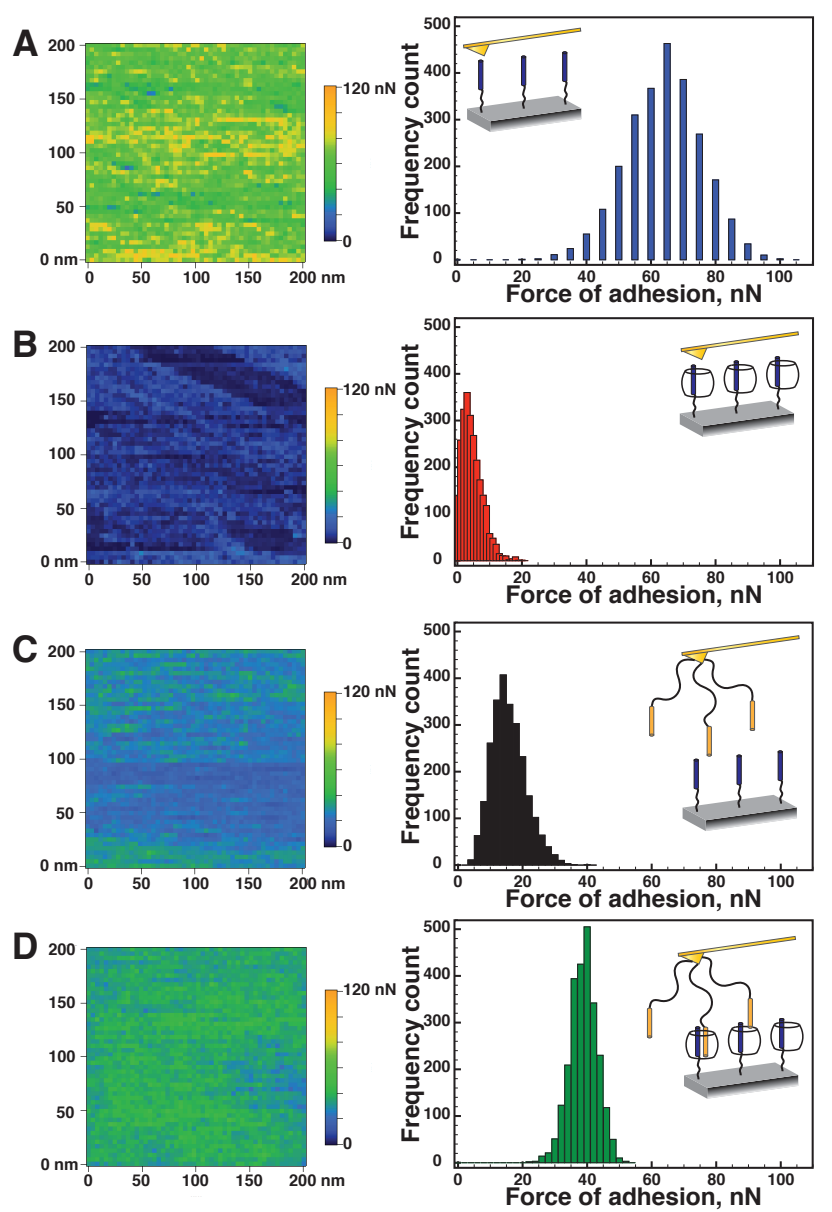

Figure 3: Adhesion mapping in fluid on MV-mica with (A) an Au tip in pure water, (B) an Azo-Au tip in pure water, (C) an Au tip in $1 \mathrm{mM} \mathrm{CB}$ [8], (D) an Azo-Au tip in $1 \mathrm{mM} \mathrm{CB[8],}$ with corresponding tip schematics and histograms of the frequency of the adhesive forces.

tip-sample interactions, resulting in a drop in the average retraction force from 65 to approximately $5 \mathrm{nN}$, Figure 3B. Figure 3C shows the interaction of the azobenzene-functionalized tip in the absence of $\mathrm{CB}[8]$. Interaction between the two guests in a non-specific fashion accounts for an increase in the average retraction force from $5 \mathrm{nN}$ to approximately $20 \mathrm{nN}$. Finally, the retraction force of the ternary complex was quantified, Figure 3D. An analysis of the adhesion map shows that the average retraction force was in the region of $40 \mathrm{nN}$. While being approximately $33 \%$ lower than the non-specific interaction of the unfunctionalized tip and mica-1 (Figure 3A), the interaction has the advantage of being fully reversible and controllable. 
A comparison of the $\mathrm{F}_{a d h}$ values arising from the macroscopic surface-surface interactions and the bulk adhesion data from force-distance spectroscopy indicate a cooperative effect as interaction strengths do not scale linearly. Bulk FDS experiments suggest the force required to remove the tip from the surface after 1 interaction is on the order of $40 \mathrm{nN}$, in contrast to the SMFS data where the $\mathrm{F}_{a d h}$ of a single interaction is only $30 \mathrm{pN}$. This would mean that 1333 heteroternary complexes must be engaged simultaneously, which is approximately $9 \%$ of the possible interactions on the area afforded by an AFM tip $(r=20 \mathrm{~nm})$ each time it makes contact with the mica-1·CB[8] surface. The non-specific adhesion peak of approximately $150 \mathrm{pN}$ must also be taken into account for this calculation (ESI, Figure S5), as it will have a large contribution to bulk adhesion. Thus, the $40 \mathrm{nN}$ value likely arises from approximately 222 cooperative interactions (only $1.5 \%$ of the total available on the AFM tip). The $\mathrm{F}_{a d h}$ of heteroternary complexation calculated from the macroscopic adhesion experiment $(1597 \mathrm{pN})$ suggests that the actual number of cooperative interactions is closer to 25 , or just $0.17 \%$ of those potentially available on the AFM tip. Clearly a small number of heteroternary interactions exhibit a substantially larger $\mathrm{F}_{a d h}$ value than would be expected if each was contributing individually. Therefore, an additional interaction is likely present.

Cooperativity appears to be important in this system, as in the natural world. It is known from Nature that several identical or complementary units engaging simultaneously lead to an overall effect that is greater than the sum of the individual linkages, e.g. Gecko feet, junctional microdomain membrane proteins and mineralized collagen fibrils in trabecular bone. ${ }^{1,27,28}$ Moreover, dissipation of stress accumulating at the mica-1/mica-2 interface must also occur, where the pulling force is distributed over many linkages in order to reduce stress on any single one. This stress dissipation increases the overall $\mathrm{F}_{\text {adh }}$ of the system and is likely mediated by the dynamic complexation/decomplexation behavior of the $\mathrm{CB}[8]$ complexes. Previous work by Appel et al. revealed that the shuttling behavior of the CB[8] and the timeframe over which this occurs is in the sub-microsecond domain. ${ }^{29}$ As long as the force exerted on the linkage does not exceed the maximum $\mathrm{F}_{a d h}$ within this timeframe, 
the linkage should reform almost instantaneously. Thus, CB[8] heteroternary complexation could serve an excellent adhesive mediator with exceptional stress dissipation properties at interfaces, as illustrated in Figure 4.

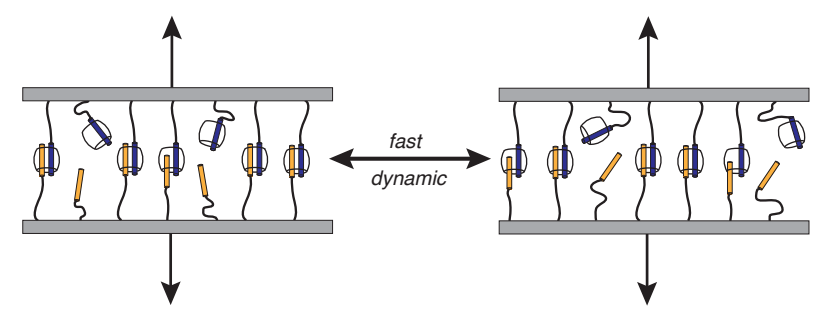

Figure 4: Idealized schematic of stress dissipation in between the mica-1 and mica-2 surfaces in the presence of $\mathrm{CB}[8]$

As noted by several researchers, adhesive strength decreases as a function of adhesive layer thickness, as such optimization of an adhesive joint focusses on enhancing adhesive strength while minimizing layer thickness. ${ }^{30,31}$ Here, the layer thickness is approximately $4.2 \mathrm{~nm}$, resulting in an observed adhesive strength over the layer on the order of $10^{11} \mathrm{~N}$, an astonishingly large value for such a thin layer, despite the extremely low surface coverage of accessible MV units (less than 0.001\%). Clearly, such thin layer small molecule systems have great potential in many application sectors such as switchable circuitry, robotics and sensor design, and in particular where stress dissipation is critical. Coupling effective stress dissipation through cooperative formation and reformation of dynamic ternary complexes at interfaces with reversibility and external photo-controlled switching is a significantly advantageous quality, which should influence the next generation of dynamic materials and functional interfaces.

\section{Acknowledgement}

We thank Dr E. Spigone for assistance with the MFP3D AFM system. PEW is grateful to the Atomic Weapons Establishment and the Melville Laboratory for Polymer Synthesis for financial support. OAS and ZWK thanks the ERC Starting Investigator Grant (ASPiRe, 
No. 240689) for financial support. OAS also acknowledges a Next Generation Fellowship provided by the Walters-Kundert Foundation. OAS and STJ acknowledge support from the EPSRC NanoSci-E+ grant, (CUBiHOLE, EP/H007024/1).

\section{Supporting Information Available}

Detailed functionalization and characterization of the mica surfaces used for these experiments is given in the supporting information. A video of the photo-reversible adhesion process is also provided.

\section{References}

(1) Ma, S.; Scaraggi, M.; Lin, P.; Yu, B.; Wang, D.; Dini, D.; Zhou, F. Nanohydrogel Brushes for Switchable Underwater Adhesion J. Phys. Chem. C 2017, 121, 8452-8463.

(2) Paretkar, D.; Kamperman, M.; Martina, D.; Zhao, J.; Creton, C.; Lindner, A.; Jogota, A.; McMeeking, R.; Arzt, E. Preload-Responsive Adhesion: Effects of Aspect Ratio, Tip Shape and Alignment J. R. Soc. Interface 2013, 10, 20130171.

(3) Rose, S.; Prevoteau, A.; Elzière, P.; Hourdet, D.; Marcellan, A.; Leibler, L. Nanoparticle Solutions as Adhesives for Gels and Biological Tissues Nature 2014, 505, 382-385.

(4) Purtov, J.; Frensemeier, M.; Kroner, E. Switchable Adhesion in Vacuum Using BioInspired Dry Adhesives ACS Appl. Mater. Interfaces 2015, 7, 24127-24135.

(5) Ko, Y.; Kim, K.; Kang, J.; Chun, H.; Lee, J.; Sakamoto, S.; Yamaguchi, K.; Fettinger, J.; Kim, K. Designed Self-Assembly of Molecular Necklaces Using HostStabilized Charge-Transfer Interactions J. Am. Chem. Soc. 2004, 126, 1932-1933.

(6) Kim, K.; Kim, D.; Lee, J.; Ko, Y.; Kim, K. Growth of Poly(Pseudorotaxane) on Gold Using Host-Stabilized Charge-Transfer InteractionChem. Commun. 2004, 40, 848-849. 
(7) Tian, F.; Cheng, N.; Nouvel, N.; Geng, J.; Scherman, O. A. Site-Selective Immobilization of Colloids on Au Substrates via a Noncovalent Supramolecular 'Handcuff' Langmuir 2010, 26, 5323-5328.

(8) Tian, F.; Cziferszky, M.; Jiao, D.; Wahlström, K.; Geng, J.; Scherman, O. A. Peptide Separation through a CB[8]-Mediated Supramolecular Trap-and-Release Process Langmuir 2011, 27, 1387-1390.

(9) Lan, Y.; Loh, X. J.; Geng, J.; Walsh, Z.; Scherman, O. A. A Supramolecular Route Towards Core-Shell Polymeric Microspheres in Water via Cucurbit[8]uril Complexation Chem. Commun. 2012, 48, 8757-8759.

(10) An, Q.; Brinkmann, J.; Huskens, J.; Krabbenborg, S.; de Boer, J.; Jonkheijm, P. A Supramolecular System for the Electrochemically Controlled Release of Cells Angew. Chem. Int. Ed. 2012, 51, 12233-12237.

(11) Gonzalez-Campo, A.; Brasch, M.; Uhlenheuer, D.; Gomez-Casado, A.; Yang, L.; Brunsveld, L.; Huskens, J.; Jonkheijm, P. Supramolecularly Orientated Immobilization of Proteins Using Cucurbit[8]uril Langmuir 2012, 28, 16364-16371.

(12) Gonzalez-Campo, A.; Eker, B.; Gardiniers, H.; Huskens, J.; Jonkheijm, P. A Supramolecular Approach to Enzyme Immobilization in Micro-Channels Small 2012, 8, 3531-3537.

(13) Tian, F.; Jiao, D.; Biedermann, F.; Scherman, O. A. Orthogonal Switching of a Single Supramolecular Complex Nat. Commun. 2012, 3, 1207.

(14) Neirynck, P.; Brinkmann, J.; An, Q.; van der Schaft, D.; Milroy, L.; Jonkheijm, P.; Brunsveld, L. Supramolecular Control of Cell Adhesion via Ferrocene-Cucurbit[7]uril Host-Guest Binding on Gold Surfaces Chem. Commun. 2013, 49, 3679-3681. 
(15) Hu, C.; Lan, Y.; Tian, F.; West, K. R.; Scherman, O. A. Facile Method for Preparing Surface-Mounted Cucurbit[8]uril-Based Rotaxanes Langmuir 2014, 30, 10926-10932.

(16) Hu, C.; Lan, Y.; West, K. R.; Scherman, O. A. Cucurbit[8]uril-Regulated Nanopatterning of Binary Polymer Brushes via Colloidal Templating Adv. Mater. 2015, 27, 7957-7962.

(17) Nakahata, M.; Takashima, Y.; Yamaguchi, H.; Harada, A. Redox-Responsive SelfHealing Materials Formed from Host-Guest Polymers Nat. Commun. 2011, 2, 511.

(18) Yamaguchi, H.; Kobayashi, Y.; Kobayashi, R.; Takashima, Y.; Hashidzume, A.; Harada, A. Photoswitchable Gel Assembly Based on Molecular Recognition Nat. Commun. 2012, 3, 603.

(19) Zheng, Y.; Hashidzume, A.; Takashima, Y.; Yamaguchi, H.; Harada, A. TemperatureSensitive Macroscopic Assembly Based on Molecular Recognition ACS Macro Lett. 2012, 1, 1083-1085.

(20) Hashidzume, A.; Zheng, Y.; Takashima, Y.; Yamaguchi, H.; Harada, A. Macroscopic Self-Assembly Based on Molecular Recognition: Effect of Linkage between Aromatics and the Polyacrylamide Gel Scaffold, Amide versus Ester Macromolecules 2013, 46, $1939-1947$.

(21) Kobayashi, Y.; Takashima, Y.; Hashidzume, A.; Yamaguchi, H.; Harada, A. Reversible Self-Assembly of Gels Through Metal-Ligand Interactions Sci. Rep. 2013, 3, 1243.

(22) Zheng, Y.; Hashidzume, A.; Harada, A. pH-Responsive Self-Assembly by Molecular Recognition on a Macroscopic Scale Macromol. Rapid Comm. 2013, 34, 1062-6.

(23) Ahn, Y.; Jang, Y.; Selvapalam, N.; Yun, G.; Kim, K. Supramolecular Velcro for Reversible Underwater Adhesion Angew. Chem. Int. Ed. 2013, 52, 3140-3144. 
(24) Walsh-Korb, Z.; Yu, Y.; Janeček, E. R.; Lan, Y.; del Barrio, J.; Williams, P. E.; Zhang, X.; Scherman, O. A. Single Molecule Force Spectroscopy Quantification of Adhesive Forces in Cucurbit[8]uril Host-Guest Ternary Complexes Langmuir 2017, 33, $1343-1350$.

(25) del Barrio, J.; Horton, P. N.; Lairez, D.; Lloyd, G. O.; Toprakcioglu, C.; Scherman, O. A. Photocontrol over Cucurbit[8]uril Complexes: Stoichiometry and Supramolecular Polymers J. Am. Chem. Soc. 2013, 135, 11760-11763.

(26) Mannan, S. Lee's Loss Prevention in the Process Industries: Hazard Identification, Assessment and Control, 3rd ed.; Elsevier, 2005; Chapter 17.

(27) Colom, A.; Casuso, I.; Boudier, T.; Scheruing, S. High-Speed Atomic Force Microscopy: Cooperative Adhesion and Dynamic Equilibrium of Junctional Microdomain Membrane Proteins J. Mol. Biol. 2012, 423, 249-256.

(28) Fantner, G.; Hassenkam, T.; Kindt, J.; Weaver, J.; Birkedal, H.; Pechenik, L.; Cutroni, J.; Cidade, G.; Stucky, G.; Morse, D.; Hansma, P. Sacrificial Bonds and Hidden Length Dissipate Energy as Mineralized Fibrils Separate During Bone Fracture Nat. Mater. 2005, 4, 612-616.

(29) Appel, E. A.; Forster, R. A.; Koutsioubas, A.; Toprakcioglu, C.; Scherman, O. A. Activation Energies Control Macroscopic Properties of Physically Crosslinked Materials Angew. Chem. Int. Ed. 2014, 53, 1-7.

(30) Gent, A. N.; Hamed, G. R. Peel Mechanics of Adhesive Joints Polym. Eng. Sci. 1977, $17,462-466$.

(31) Carpinteri, A.; Pugno, N. Are Scaling Laws on Strength of Solids Related to Mechanics or to Geometry? Nat. Mater. 2005, 4, 421-423. 


\section{Graphical TOC Entry}

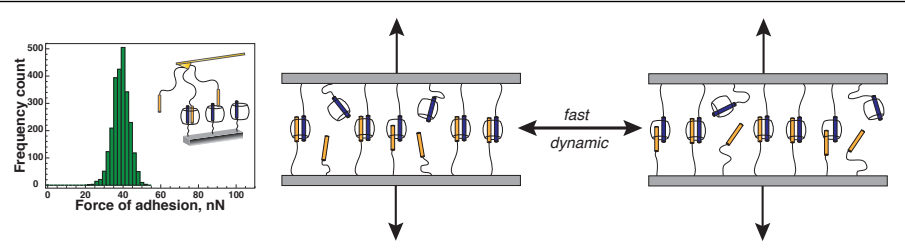

Comparison of single molecule, bulk and macroscopic adhesion behavior give insight into cooperativity and stress dissipation in dynamic adhesive systems. 